\title{
Research on the Tracking Evaluation of Learning of Vocational College Students in School
}

\author{
Wei $\mathrm{Ma}^{1, \mathrm{a}}$ \\ ${ }^{1}$ Qinhuangdao Institute of Technology ,China,066100 \\ aqhdmv@126.com
}

Keywords: Vocational College Students, Tracking Evaluation, Learning

\begin{abstract}
Through the investigation on vocational college students' learning during school, this paper analyzed the characteristics of vocational college students' learning from teachers "teaching" perspective and students "learning" perspective, discussed the way for vocational colleges students to improve learning ability and learning interests. Only improve student learning initiative, in order to make it consistent with the needs of society and to obtain theoretical knowledge and practical skills to meet the needs of social and economic development.
\end{abstract}

\section{Introduction}

Vocational college students in general are high school graduates and previous graduates. Due to the different objects, knowledge of its students constitutes a large difference in knowledge of high school graduates coherence. and the foundation are better, but the skills are weak; strong "three schools 'graduates' skills capacity, but some of the basics weak, junior high school graduates have the knowledge and skills in the culture stage, not yet fully apparent faced with such a complex structure of students, higher vocational colleges face enormous pressure, but the pressure can be converted to large power vocational education despite the difficulties, but to overcome the difficulties and obstacles on the road ahead, we are bound to usher a bright future.

\section{The Learning of Students in Higher Vocational Colleges}

Classroom Learning. Classroom learning is the flexibility and can change the teaching sites dynamically according to actual needs. Regardless of the specific needs and learning styles as long as the teaching situation mate, as schools and teachers should be based on their actual conditions and the ability to meet such conditions for learning. The reason for making such a view because the vocational and technical education and general basic education are two entirely different types. Their reasons are based on the theory related to vocational education on what is not groundless.

In short, I believe that the classroom can not be ignored include four features. It must have participation of students and teachers, there must be a certain learning objectives. There must be a learning environment, there must be an evaluation of learning systems involved. If there is no such limit of four, it is not our classroom to be discussed.

Cultural Courses. Culture courses generally take the form of classroom in the school and the traditional forms of classroom learning. Professional theory courses in general use school classrooms or what we call the second classroom training workshop and the workshop so that the two companies to expand the teaching spaces, also belong to the category of classroom learning. Culture of learning in students' knowledge mainly refers to the degree of mastery of the school curriculum. Quality standards are generally uniform national regulations. But textbooks or traditional subject knowledge cultural studies centered model, not a good combination to expand vocational education, this may be the need to reform the curriculum of local culture. Cultural lesson in vocational school learning difficulty faced by learning as much as the other two courses. Because students general culture courses vocational school than the poor, it was "examination-oriented education" eliminated, forced into the vocational school learning.

Theory Professional Courses. Professional theory courses are essentially within the wisdom of learning skills. You can learn which finds inspiration in the wisdom of Gagne skills. This is not 
done detailed analysis. As a professional course of study quality evaluation of teachers is the most qualified to speak.

Practice Courses. Practical training courses or study or be referred to hands-on learning is that students learn in "real knife real gun," the professional environment, which is an important aspect of the quality of student learning. Or it can be said that practical learning is a critical stage of forging professional quality of students, and evaluate the quality of learning is more important at this stage. Practice course is a comprehensive study. Because the three courses in vocational and technical education, vocational and technical education as a feature of the course is practical courses, vocational and technical education or the education and the general is no different.

\section{The Analysis of Vocational College Students‘ Learning in School}

The learning emotion is burnout and learning efficiency is low. Students in vocational colleges have promoted over large undergraduate studies, but due to lack of college exam or learning ability, this rise suffered failure. Vocational College, one of the teachers complain about students learning or inert low starting point, the other students complained courses boring or useless, school teachers, lack of passion as chanting, students in the course of showing the weariness. Because of the relative lack of knowledge for the deletion good study habits, many students encounter difficulties in vocational learning phenomenon appeared agitated. Courses to learning phase, a considerable portion of students even fear emotions, people do not like to study and in-depth study, few can enjoy learning to solve problems after the pleasure, on the contrary, too much experience to be underachieving good learning inefficient hardship. And future employment pressure, many students despair, loss of confidence in the ideological mood mostly negative, impetuous, anxiety, tension, depression, self-denial and other state, these negative emotions directly affect students' learning behavior and learning processes.

They lack of self-confidence to improve academic performance, learning behavior and persistent lack of consciousness. From students to apply to the coal and professional views on vocational education, most students entering vocational colleges have inferiority complex, in conversation with students in a clear sense that they and their own admiration for the students admitted to colleges defeat invisible angry. You can see from the behavior of some students study and life, they often put their loss attributed to the external environment, rather than their own lack of effort. When teachers encourage them to study diligently, there is still hope that we can continue to learn when entering undergraduate institutions realize their ideals, and most students tend to show a lack of confidence, that their case, limited prospects for further efforts.

They lack of enthusiasm for learning, lack of healthy life after school. Vocational students are relatively weak in ability to analyze problems' thinking and the relative lack of refining capacity. Although the majority of students desire to learn, but at the same time they are mentality impetuous, lack of enthusiasm for learning, improper learning methods. Like problems with learning interesting, boredom requires in-depth understanding and analysis of learning content, training and situational practice preferences. Lack of theoretical knowledge and learning motivation and interest, just about understanding, showing the image of the thinking ability, abstract thinking inadequate level features. Investigation showed that except for a small number of vocational students have a hobby or have expertise in art, calligraphy, sports and other aspects, there are a considerable number of students after-school life more simple, more for playing online games, chatting, watching romantic novels and so on. School life is monotonous, lack of health, positive, progressive activity or hobby.

\section{The Countermeasures}

Setting reasonable or timely adjustment of curriculum and teaching programs. Higher vocational education is to train practical skills and technical personnel for the purpose. Therefore, higher vocational education should be practical. On the one hand, enterprises should be in-depth site workplace research to understand the development of science and technology, the current job market has been abandoned, obsolete technology and equipment conditions; on the other hand, 
graduates of feedback information through the old track the most modern production site What is needed is the ability to direct, students in vocational colleges should have the ability to adapt to what the workplace needs, and then set a reasonable or timely adjustment of curriculum and teaching programs.

Guide students to establish a correct goal in life, to encourage students to strive for their own development. Vocational students in thinking is not mature enough, attitude towards life and psychological mechanisms are also in the formative stages, the pursuit of stimulation wave of bad social influence their thinking correct values and outlook on life. Therefore, strengthen ideological education, clean and optimize the minds of students, guide students to establish a correct goal in life, to help students grow up healthily is particularly important. Survey shows that due to the intense study in high school and college entrance examination of the experience of failure liter, Vocational Students make sense and sense of failure slack exists in varying degrees, with a clear irritability and boredom set in the study. Teachers should help students overcome this mood, to encourage them to strive for their own healthy development. Teachers should be integrated into the student population, full communication with students as equals, standing on the point of view of students and students to discuss equality, understanding students' learning experience and learning puzzles, and give them timely counseling and guidance. Through classes, seminars, lectures and outings, and other forms of learning activities, issues of interest to the students from the start, and students to discuss how to make their own future is full of color, students positive life imperceptibly attitudes and learning to guide students to establish a correct goals in life, to enable students to understand the significance of the realization of personal value.

Enhance the mental health education to help students to enhance self-confidence. Vocational students learning in general are awareness, a lack of ability to learn more. When they have worked so hard but no significant effect, it will have a psychological frustration, helplessness and boredom learning, resulting in depressed students' lack of confidence, emotional instability, lack of persistence, and many other learning psychological problems. While teachers are teaching, should pay attention to guide students to learn psychology trends, strengthen the relevance of mental health education.

Teach students how to learn, students self-learning ability. Surveys show that a considerable number of vocational students are not really grasp the learning methods courses, blindly follow the exam-oriented education model, strong psychological dependence, teacher scholarship, teachers do not see what assignments do not know 'what to review. There are a considerable number of vocational students do not study notes, the learning process is limited to the classroom, not self, does not use the library access to relevant information in order to deepen the understanding of professional knowledge and professional competence and mastery of consolidation. There educators believe that if students can take the initiative in learning to generate links between knowledge, he will form a deeper and better understanding.

Stimulate students' interest in learning, teaching master difficult to develop meaningful tasks. To stimulate students' interest in knowledge is the key to get rid of weariness. Studies have shown that too difficult or too easy to learning tasks are conducive to the generation of interest in learning. Therefore, the correct grasp of teaching difficulty, a reasonable allocation of teaching time, moderately reduced learning curve, reducing the learning process of frustration, restore confidence so that students' interest in learning to improve critical.

Teachers should be good at discovering and exploiting student curiosity, the passive "filling" becomes active students "learning." Guide students to actively think and be timely praise and encouragement, students complete tasks in the process of improving students' sense of participation and confidence. Specialized in teaching, we often find that there are usually parts of the poor performance of students in class, once went to the training laboratory, enthusiasm greatly improved, always to the many questions posed by the teacher. Thus, if in the course of teaching the theory, practice and more through the links, and more practice, production practice teaching courses conducted in the field of actual cases, students are given cases of doubt and eager to know the answers and results, the allowing them to find the answer or solution to the problem given measures 
and programs to fully mobilize their learning, explore the enthusiasm and attention, will make them increasingly turning their attention to the study, and the sustained attention and thinking.

\section{Conclusions}

To get rid of the vocational college students learning burnout and other negative emotions during the school we should find an effective way to learn from both teaching and learning. From "all for student development" and "people-oriented" point of view, to take positive and effective education measures, correct the deviations of educational concepts and teaching behavior; secondly, to make reasonable guide for students to establish a correct worldview and values in teaching.

Acknowledgement

This paper is the special research results of the 2015 National Vocational Education Quality Assurance and Assessment and the project name is "The Research on the Learning Tracking Evaluation of Students in School Based on Student"

\section{References}

[1] Huifang Zhou: Vocational and Technical Education, Vol. 6 (2004) No 53, p.25-26

[2] Yu Yan: Exploration and Research on Vocational Education, Vol. 12 (2005) No 27, p.74-76

[3] Qin Guo: Technical Education Forum, Vol. 1 (2006) No 33, p.11-14

[4] Jieming Liu: Modern Enterprise Education, Vol. 3 (2007) No33, p.121-124

[5] Yanli Liu: Communication of Vocational Education, Vol. 12 (2012) No23, p.57-64 